\title{
Initiatives en Matière de Gestion des Déchets Solides Municipaux (DSM) : Vers l'Emergence d'une Economie Verte dans la Ville de Dschang, Cameroun
}

\author{
Joël Sagne Moumbe, MA \\ Aristide Yemmafouo, PhD \\ Maurice Tsalefac, PhD \\ Leon Josiaste Fapong, MA \\ Département de Géographie, Université de Dschang, Cameroun
}

Doi:10.19044/esj.2020.v16n14p123 URL:http://dx.doi.org/10.19044/esj.2020.v16n14p123

\section{Résumé}

La gestion des déchets est une préoccupation majeure pour la ville de Dschang qui ne bénéficie pas du service d'enlèvement par HYSACAM ${ }^{4}$, l'opérateur privé présent dans d'autres villes et subventionné par l'Etat. Pour faire face à ce problème, la ville a mis sur pied plusieurs initiatives grâce à la coopération décentralisée et au partenariat public-privé. Pour mettre en évidence l'impact qu'ont ces initiatives dans la construction d'une économie verte, une enquête a été conduite auprès de 269 ménages, 61 commerçants, 52 utilisateurs du compost, 20 composteurs. Des données relatives à l'activité de précollecte, à la production du compost, sa commercialisation et au monitoring carbone ont été collectées auprès de l'Agence Municipale de Gestion des Déchets (AMGED). Le traitement statistique et l'analyse thématique des données quantitatives et qualitatives révèlent la mise en place, à partir de la gestion des déchets à Dschang, d'activités génératrices de revenus et contribuant à la protection de l'environnement. Avec $79 \%$ des ménages qui paie le service à la fin du mois, l'activité de précollecte a permis à une structure de tendre vers l'équilibre financier. Grâce à la précollecte participative, les quartiers peu accessibles sont désormais intégrés. La transformation des déchets biodégradables en compost permet d'éviter les émissions des gaz à effet de serre, d'avoir un intrant pour l'agriculture locale et des recettes issues de sa vente. Le compostage communautaire qui met en commun plusieurs ménages, redynamise le lien social. Au total, 80 emplois verts ont été créés pour un revenu minimum mensuel de 40000 FCFA soit 60 euros.

\footnotetext{
${ }^{4}$ Hygiène et Salubrité du Cameroun
} 
Mots clés : Compostage, déchets solides municipaux, économie verte, précollecte participative, Dschang, Cameroun

\title{
Municipal Solid Waste (MSW) Management Initiatives and Design of a Green Economy Strategy in the City of Dschang
}

\author{
Jö̈l Sagne Moumbe, MA \\ Aristide Yemmafouo, PhD \\ Maurice Tsalefac, PhD \\ Leon Josiaste Fapong, $M A$ \\ Département de Géographie, Université de Dschang, Cameroun
}

\begin{abstract}
Waste management is a major concern for the town of Dschang who are not benefiting from the HYSACAM collection services, a private service operating in other cities and subsidized by the State. In addressing this problem, the city has employed several initiatives through decentralized cooperation and public-private partnership. This paper focuses on highlighting the impact of these initiatives in the building of a green economy. To achieve this, a survey was conducted on 269 households, 61 traders, 52 compost users, and 20 composters. Data relating to pre-collection activity, compost production, marketing, and carbon monitoring was collected from the Municipal Waste Management Agency (MWMA). Statistical processing and thematic analysis of quantitative and qualitative data revealed the income generating activities and environmental protection through waste management in Dschang. With a coverage rate of $79 \%$, the participatory pre-collection has generated an operator that tends towards financial autonomy. Due to participatory pre-collection, inaccessible neighbourhoods are now integrated. Composting organic waste reduces greenhouse gas emissions, generates revenue, and produces organic manure for the development of local agriculture. Community composting revitalizes social ties. In total, 80 green jobs were created for a minimum monthly income of 40,000 FCFA or 60 Euros.
\end{abstract}

Keywords: Composting, municipal solid waste, green economy, participatory pre-collection, Dschang, Cameroon 


\section{Introduction}

La gestion des déchets ménagers constitue un défi majeur dans les villes des Pays En Développement (PED) où les municipalités doivent faire face à des quantités croissantes de déchets, avec des moyens financiers limités (Naquin et al., 2008). L'accroissement de la production des déchets ménagers est lié la forte croissance démographique et à l'urbanisation non maitrisée dans les pays du sud (Thonart et al., 2005). Les récents chiffres de la Banque mondiale sur la production et les modes de gestion des déchets en Afrique Subsaharienne montrent que la situation de l'insalubrité urbaine tend à s'empirer. En effet, seulement 44\%, soit moins de la moitié des déchets produits sont collectés (Kaza et al., 2018). Au Cameroun, pour renforcer sa politique de gestion des déchets, l'Etat a revu le cadre juridique et institutionnel, transférant les compétences aux communes dans le cadre de la décentralisation. Cependant l'absence de transfert total des ressources en faveur des acteurs locaux fait plutôt croire à une décentralisation de la pénurie comme le souligne Otayek (2009). Dans ce contexte, la ville de Dschang, avec l'appui technique et financier de nombreux partenaires nationaux et internationaux développe et met en œuvre des initiatives depuis le milieu des années 2000. Le but étant de faire participer les populations et tous les acteurs en visant à terme la construction d'une stratégie locale d'assainissement solide. Ces initiatives sont la précollecte participative, le compostage et le monitoring carbone. La question est donc de savoir comment ces différentes initiatives en matière de gestion des déchets solides municipaux (DSM) contribuent à la mise en place d'une stratégie d'économie verte ?

L'objectif est donc de mettre en évidence l'impact de ces initiatives dans l'émergence d'une économie verte à Dschang. Il est postulé que la décentralisation de la gestion des DSM permet l'éclosion des initiatives de coopération multilatérale de proximité permettant de mettre sur pied des projets viables. Pour comprendre les initiatives d'économie verte dans la gestion des DSM à Dschang, il est démontré la maitrise progressive des impacts environnementaux (1), les retombées économiques intégrées dans le développement local (2), et surtout les implications dans l'amélioration des conditions de vie (3). Une discussion permet enfin de relever l'originalité de l'expérience de Dschang qui semble aujourd'hui faire cas d'école au service du rayonnement national et international de la ville.

\section{Méthodologie}

Etalée sur une superficie de 5655 ha (POS 20105), Dschang, ville moyenne d'une population de 101124 (RGPH, 2005) habitants est située dans l'arrondissement qui porte le même nom et limitée au Nord et au Nord-Ouest

${ }^{5}$ Commune de Dschang, Plan d'Occupation des Sols, 2010 
par l'Arrondissement de Fongo-Tongo, à l'ouest et au Sud-Ouest par l'Arrondissement de Santchou, au Sud et au Sud-Est par l'arrondissement de Fokoué, à l'Est par l'arrondissement de Nkong-Ni (Figure 1). Elle est blottie à $1400 \mathrm{~m}$ sur le versant Sud-Est des monts Bamboutos (2740m) entre $5^{\circ} 25$ et $5^{\circ} 30$ de latitude Nord, et $10^{\circ} 30$ et $10^{\circ} 50$ de longitude Est.

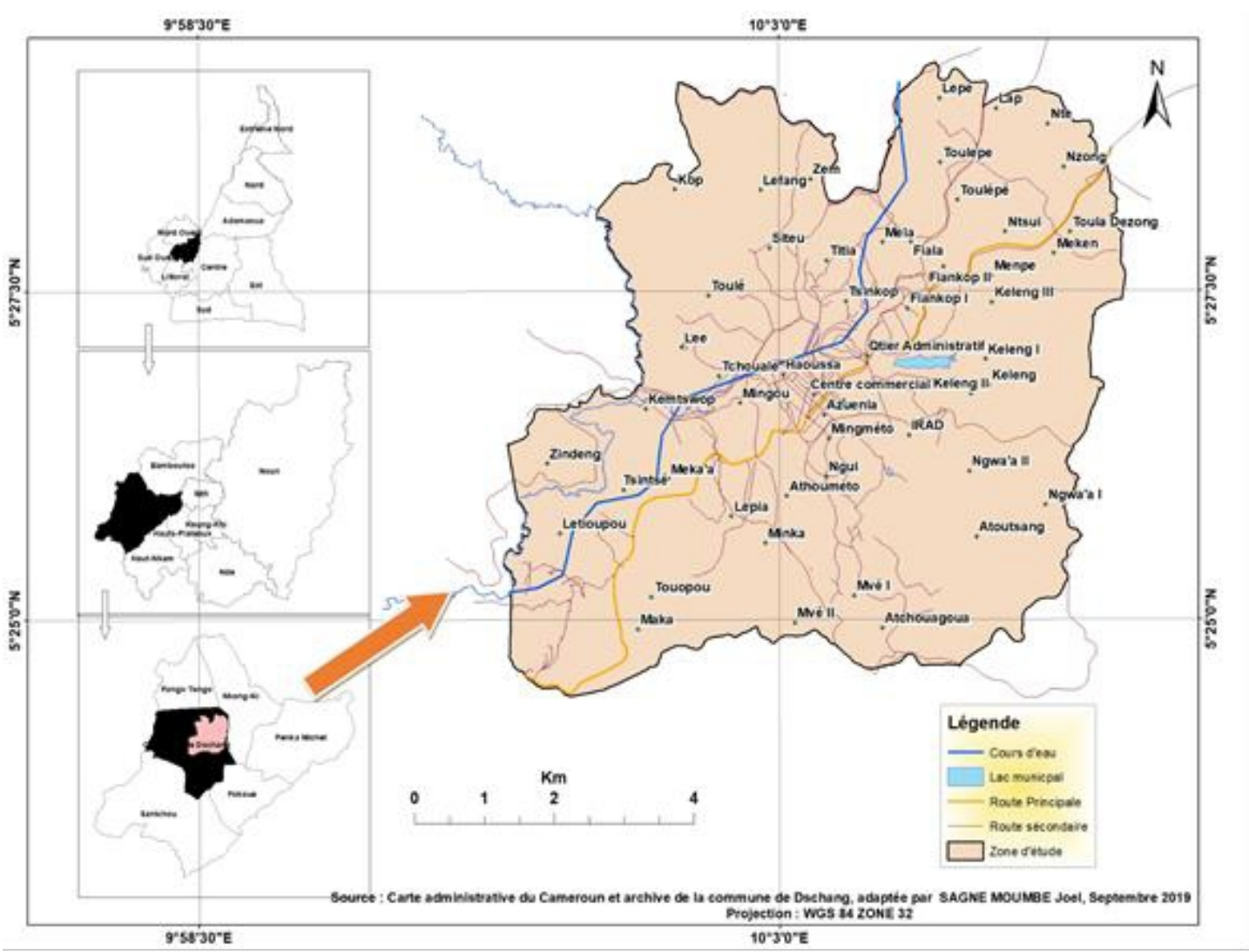

Figure 1. Situation de la zone de recherche

Une enquête par questionnaire auprès de 269 ménages, 61 commerçants, 52 utilisateurs du compost, 20 composteurs et 21 entretiens effectués pendant les années 2017 et 2018 a permis d'obtenir les données. Pour l'enquête ménage, la ville a été découpée en trois secteurs socioéconomiques sur la base des données du POS : les habitats moyen standing, la zone commerciale (les marchés), les habitats bas standing. L'échantillonnage aléatoire systématique a été utilisé pour choisir les personnes à enquêter. Un pas dégressif de 6,3,1 a été retenu selon la densité des ménages le long des axes de circulation. Dans les marchés, les commerçants ont été enquêtés selon le type de produits vendus. L'échantillonnage aléatoire stratifié a été utilisé pour choisir parmi les petits utilisateurs de compost (moins de 2 tonnes), les utilisateurs moyens (entre 3 et 5 tonnes) et les grands utilisateurs (plus de 5 
tonnes), ainsi que les composteurs. Les entretiens ont été menés auprès du personnel de l'Agence Municipale de Gestion des Déchets (AMGED (7), des structures de précollecte (6), des agriculteurs bio (5) et des clients des produits bio (3). Les données relatives à l'activité de précollecte, à la production-vente du compost, et au monitoring carbone ont été collectées auprès de l'AMGED.

La précollecte participative ${ }^{6}$ consiste à la collecte porte-à porte des déchets par des organisations de la société civile (OSC) à une fréquence moyenne de trois fois par semaine contre un paiement mensuel variable selon les zones géographiques et les standings des habitations : (500 à $2000 \mathrm{Fcfa}^{7}$ selon la taille des ménages et 100 Fcfa pour les chambres d'étudiants. Ces OSC couvrent donc tous les quartiers de la ville y compris les moins accessibles puisqu'elles opèrent avec des tricycles et sous un régime de partenariat publicprivé très compétitif.

Le compostage sur plateforme et dans les communautés résidentes, développé depuis 2011, permet de rendre le compost disponible pour tous les agriculteurs de la ville et ses environs. Le monitoring carbone qui est le suivi des paramètres de calcul des réductions d'émissions au cours du processus de compostage permet l'estimation des émissions évitées à vendre au marché de compensation volontaire. Ces réductions sont obtenues en faisant la différence entre les émissions de référence qui se seraient produites si les déchets avaient été mis directement dans le site d'élimination des déchets et les émissions du projet qui sont produites à la fois par le processus de compostage et par l'électricité utilisée sur la plate-forme. Plus de deux années d'observations de terrain, notamment dans les parcelles expérimentales mises en place par l'AMGED ont permis de relever les analyses qualitatives. Le traitement des données quantitatives a été fait à l'aide de SPSS et restituées sous forme de tableaux descriptifs tant dis que les données qualitatives ont fait l'objet d'analyse thématique.

\section{Résultats}

\subsection{Des impacts environnementaux de plus en plus maitrisés} Amélioration de la salubrité et réduction des inondations dans la ville

Les initiatives développées dans la ville de Dschang en matière de gestion des déchets ont permis d'intégrer et d'améliorer considérablement l'état de salubrité dans les quartiers à accès difficile comme Ngui, Mingmeto jadis réputés pour leur insalubrité. Le Tableau 1 présente le ressenti des populations dans les quartiers où l'activité de pré collecte s'est implantée depuis deux ans. La réduction des dépôts sauvages 97\%, l'amélioration de la propreté (96\%) et la

\footnotetext{
${ }^{6}$ Il s'agit en fait d'une précollecte payante. « Participative » est un euphémisme utilisé pour atténuer l'idée du paiement et susciter l'adhésion d'un grand nombre de ménages au service

${ }^{7} 1$ euro $=655,957$ FCFA
} 
diminution des inondations (94\%) ont été les premiers résultats indéniables des actions de la précollecte.

Tableau 1. Impacts de la précollecte sur la qualité de l'environnement dans les quartiers à accès difficile

\begin{tabular}{|l|l|l|l|}
\hline \multirow{2}{*}{ Eléments concernés } & \multicolumn{3}{|c|}{ Avis des populations } \\
\cline { 2 - 4 } & Positif & Négatif & Neutre \\
\hline Réduction des odeurs & $84 \%$ & $2 \%$ & $14 \%$ \\
\hline Réduction de la pollution visuelle & $85 \%$ & $5 \%$ & $10 \%$ \\
\hline Baisse des maladies & $70 \%$ & $15 \%$ & $15 \%$ \\
\hline $\begin{array}{l}\text { Diminution des bêtes dans les } \\
\text { maisons } \\
\text { (Cafards, souris, moustiques, etc.) }\end{array}$ & $74 \%$ & $4 \%$ & $22 \%$ \\
\hline $\begin{array}{l}\text { Amélioration de la propreté } \\
\text { dans le quartier }\end{array}$ & $96 \%$ & $1 \%$ & $3 \%$ \\
\hline Réduction des dépôts sauvages & $97 \%$ & $2 \%$ & $1 \%$ \\
\hline Reduction des inondations & $94 \%$ & $0 \%$ & $6 \%$ \\
\hline $\begin{array}{l}\text { Curage des caniveaux et sections } \\
\text { de cours d'eau }\end{array}$ & $75 \%$ & $10 \%$ & $15 \%$ \\
\hline $\begin{array}{l}\text { Réduction des incinérations à ciel } \\
\text { ouvert }\end{array}$ & $80 \%$ & $7 \%$ & $13 \%$ \\
\hline
\end{tabular}

Source : AMGED, enquêtes de terrain, Juillet 2018

\section{Réduction des Gaz à effet de Serre (GES)}

Le compostage des ordures ménagères se fait par biodégradation des déchets fermentescibles en présence d'oxygène. Cette dégradation aérobie, contrairement à la fermentation anaérobie dans les décharges, ne produit pas de méthane. La dégradation aérobie de la matière organique produit de la vapeur d'eau, du gaz carbonique (émission compensée par la quantité de CO2 consommée par les plantes lors de la photosynthèse) et de la chaleur. Une partie de l'azote organique et minéral est convertie en azote gazeux mais ce dernier n'étant pas un gaz à effet de serre, il n'a pas d'effet sur le réchauffement global. Par rapport à la mise en décharge, le compostage permet donc de réduire les émissions de gaz à effet de serre. Dans les conditions de la ville de Yaoundé, les travaux ont montré que le compostage, par rapport à la mise en décharge classique, permet une réduction de 1,77 t éqCO2 (équivalent $\mathrm{CO} 2$ ) par tonne d'ordure ménagère (Ngnikam et al., 2002). Très faiblement mécanisé, comme c'est le cas dans la ville de Dschang, le compostage consomme très peu d'énergie et émet par conséquent très peu de $\mathrm{CO} 2$. Les réductions d'émission de gaz à effet de serre qui en résultent sont importantes. La Figure 2 montre la quantité des GES évités, le scénario de référence et les émissions du projet, par tonne de déchets traités au cours de l'année 2018 dans la ville de Dschang. 


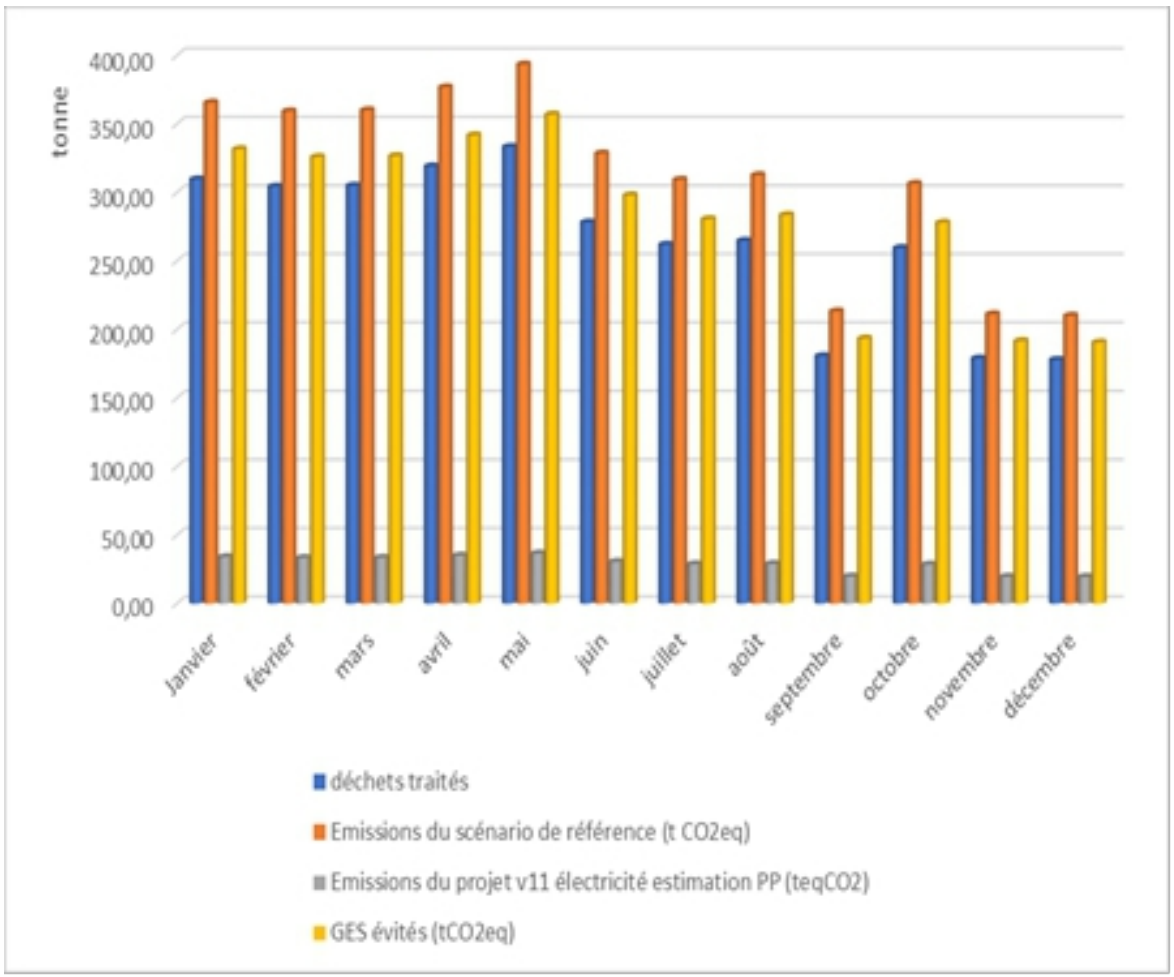

Figure 2. Quantité de GES évités en fonction du tonnage traité des déchets Source : Enquêtes de terrain, février 2019

Il ressort de cette figure que 3174 tonnes de déchets traités par compostage en 2018, ont permis d'éviter 3399 tonnes éqCO2 de s'émettre vers l'atmosphère. Les émissions du scénario de référence sont de 3747 tonnes éqCO2 et les émissions du projet de 348 tonnes éqCO2.

\section{Amélioration des sols}

La dégradation de la qualité physique des sols est un problème bien connu dans la région de l'Ouest pendant et après la caféiculture. L'altération de la structure du sol pose à la fois des problèmes d'ordre agronomiques (circulation de l'eau et des gaz, enracinement des plantes, propagation des agents pathogènes) et environnementaux (érosions). La stabilité de la structure des sols argilolimoneux, limono-sableux, qui dépend des teneurs et de la dynamique des matières organiques, est un des déterminants de la dégradation physique de ces sols allant de la formation de croûtes de battance aux risques d'érosion hydrique (Annabi, 2005). Une grande proportion de ces sols a des teneurs faibles en matières organiques. En haut de montagne, sur sol ferrallitique, un essai a été effectué avec le compost produit à Dschang, l'objectif étant de voir comment le compost se comporte comme agent agrégeant les particules de sol et de ce fait protecteur contre l'érosion. Ces travaux réalisés par Beyeme (2011) ont montré 
que plus la teneur en compost est élevée (0 tonne/ha, 10, 20 et 30 tonnes/ha) plus le diamètre moyen des agrégats est gros et plus le sol est stable. Il est observé une légère augmentation du diamètre des agrégats (passant de 0,0671 à 0,0688 mm) avec l'application du compost et davantage avec les doses croissantes de compost $(10,20$ et 30 tonnes/ha) ; ceci a été attribué à la reprise de l'activité biologique dans le sol après incorporation du compost.

Le compost produit est assez riche en substances humiques et constitue un excellent produit d'amendement des sols. Il permet à la fois d'améliorer les propriétés physiques, chimiques et biologiques du sol et de fournir par voie de minéralisation des éléments nutritifs assimilables par les plantes cultivées (Soudi, 2001). Le premier intérêt des amendements organiques issus des déchets ménagers est donc une diminution de la part des engrais chimiques lixiviables et leur remplacement par des déchets organiques valorisés. Cette réduction est de $25 \%$ chez les petits utilisateurs de compost (moins de 2 tonnes/an), 35\% chez les utilisateurs moyens (entre 3 et 5 tonnes/an) et $50 \%$ chez les grands utilisateurs (plus de 5 tonnes/an). Suivant les propriétés générales du compost des déchets ménagers, et les différentes expérimentations menées, à partir du compost produit à Dschang, son utilisation comporte plusieurs avantages pour les sols, notamment : (1) L'amélioration de la porosité, de la capacité de rétention d'eau et de la structure du sol. L'activité microbienne est essentielle à la porosité du sol. Les micro-organismes décomposent les matières organiques pour rendre les nutriments accessibles aux végétaux. Le compost étant constitué de particules de tailles différentes, il offre une structure poreuse qui améliore la porosité du sol. (ADEME, 2008 ; ADEME, 2001). (2) Des effet sur les caractéristiques physicochimiques du sol. En se minéralisant, le compost fournit des substances nutritives progressivement assimilables par les plantes. La matière organique du compost améliore les propriétés physiques du sol, augmente son pouvoir tampon. De nombreuses études ont montré le rôle bénéfique du compost sur les qualités physiques et chimiques des sols amendés. Par exemple, une amélioration des propriétés physiques; une augmentation de la conductivité hydrique et une diminution de la densité des sols ont été observées par Wong et al. (1999). De même, l'incorporation de compost au sol s'avère efficace pour lutter contre la dégradation de la surface du sol (Bresson et al., 2001). Pagliai et al. (2004) ont montré que l'ajout de compost dans un sol améliore sa porosité et sa structure. Les amendements en matière organique stable augmentent le pouvoir tampon et la capacité d'échange des sols, deux paramètres qui conditionnent la nutrition minérale des plantes (Mustin, 1987). Le compost mûr évite une acidification du sol, corrige ou réduit son acidité et diminue ainsi les risques d'exportation des métaux vers la plante (Bolan et al., 2003). Les retombées économiques sont encore plus encourageant. 


\subsection{Des retombées économiques intégrées dans le développement local Création des entreprises d'économie sociale}

Lors de la mise en place de l'activité de précollecte participative à Dschang, l'option choisie était un tiers rémunéré. Dès 2016, la municipalité après appel à manifestation d'intérêt a recruté deux associations (CEPDEL, TOCKEM), puis une troisième (ADECOTEC) en 2017 qui récupèrent contre paiement d'une rémunération, les déchets des ménages à domicile. Les montants de rémunération varient : 100 FCFA pour une chambre d'étudiant, 500 FCFA pour un ménage de taille moyenne de 5 personnes, entre 2000 et 5000 FCFA par mois pour les hôtels. Ces prix ne sont pas très différents de ceux observés dans la ville de Yaoundé (Ngambi, 2008). L'exploitation des bilans de réalisation des trois structures de précollecte permet de faire les analyses suivantes: (1) Deux des structures ont une moyenne mensuelle de 400 abonnés en 2018 et une de 500 pour un cumul annuel total de 1472 abonnés dans la ville. Chaque structure dispose de deux tricycles pour le service dans sa zone d'activité. (2) Les taux de recouvrement mensuel sont situés entre $28 \%$ et $85 \%$ avec une moyenne générale de $65 \%$, pour une recette annuelle totale de 5850250 FCFA en 2018.

Il est ressorti des enquêtes de terrain, que la qualité du service influence fortement le taux de recouvrement pour les abonnés au paiement mensuel. Les abonnés qui ont souscrit et payé pour un an expriment leur niveau de satisfaction par le renouvellement de l'abonnement ou la résiliation du service en fin d'année. Les sommes recouvrées permettent aux structures de supporter les charges courantes que sont : l'entretien et réparation des tricycles, l'achat du carburant, le paiement des salaires, des frais de communication et la constitution d'un fonds d'amortissement pour le renouvellement des tricycles. La Figure 3 présente le bilan mensuel (recettes-charges) par opérateur de pré collecte. 


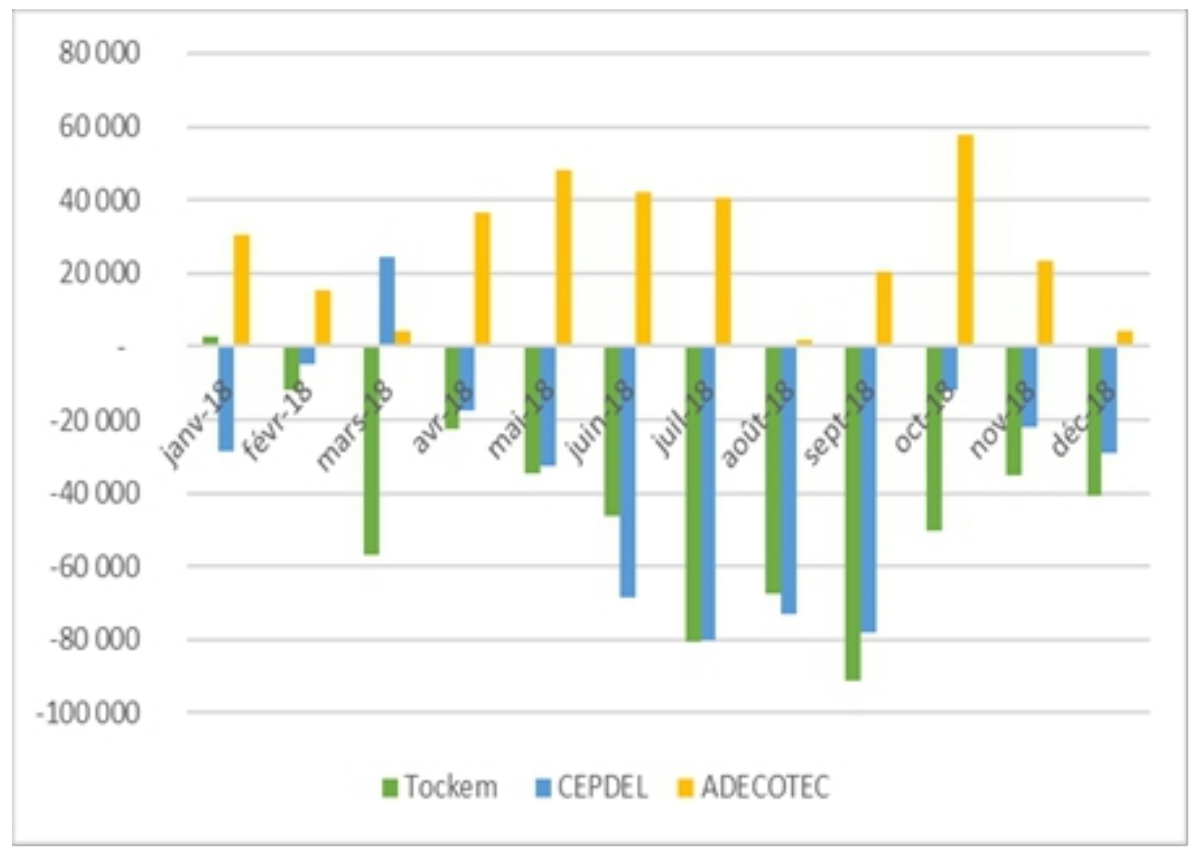

Figure 3. Bilan mensuel par structure de pré-collecte

Source : AMGED, enquêtes de terrain, Janvier 2019

Le bilan général de l'activité en 2018 est déficitaire de 579200 FCFA, mais de manière particulière, la structure ADECOTEC qui dispose d'un cumul annuel de 469 abonnés et qui a le plus grand taux moyen de recouvrement $79 \%$ a un solde annuel de 325275 FCFA dont une partie a été utilisée pour le paiement du loyer et l'autre pour l'équipement. Une simulation des recettes des deux autres structures déficitaires à partir du taux moyen de recouvrement atteint par la structure présentant un bilan positif montre que la précollecte est une activité économiquement viable.

\section{Développement agricole}

Bien qu'en faibles quantités, le compost apporte des éléments minéraux aux plantes. Couplé aux effets positifs de la matière organique, cela permet d'améliorer le rendement des cultures. Des essais d'ajout de compost sur des cultures ont montré, en fonction des sites, une amélioration du rendement pouvant aller de 50 jusqu'à $100 \%$. Cette amélioration des rendements permet d'augmenter les revenus des agriculteurs et de réduire les efforts à déployer pour le défrichement de nouvelles terres agricoles (Naquin \& Ngnikam, 2008). Suivant la dose de 15 tonnes/ha vulgarisée par l'AMGED), 1247 tonnes de compost ont été vendues par la ville de Dschang entre 2014 et 2018. Cette quantité a permis de développer l'agriculture sur près de 249 ha, avec utilisation exclusive du compost dans d'autres parcelles pour la promotion de l'agriculture biologique. 
Les quantités de compost utilisées par culture ont été standardisées pour les sols locaux (Figure 4).

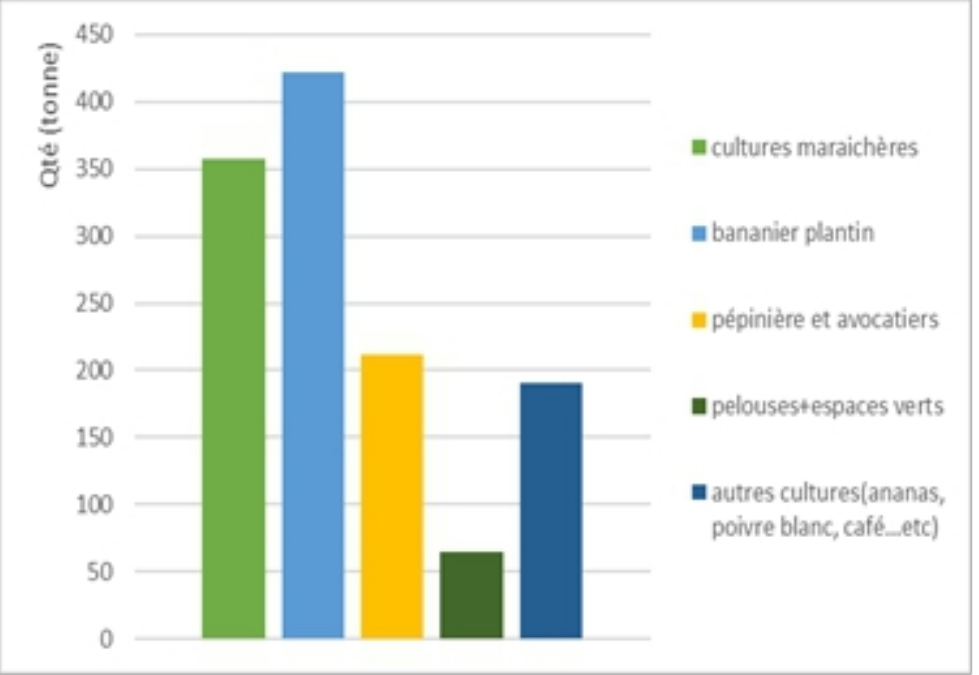

Figure 4. Utilisation du compost suivant les cultures Source : Enquêtes de terrain, février 2019

$33 \%$ du compost acheté dans la ville de Dschang a été utilisé pour la culture du bananier plantin, $28 \%$ pour les cultures maraichères, $16 \%$ pour les pépinières et la création des vergers, $15 \%$ pour les autres cultures à savoir l'ananas, le poivre blanc, le café. Les agriculteurs ayant utilisé le compost pour la caféiculture appartiennent au réseau de la Coopérative Agricole des Planteurs de la Menoua (CAPLAME) qui intervient dans la vulgarisation du compost. 5\% de compost utilisé pour les pelouses, représente la quantité achetée pour l'aménagement de l'aire de jeu du stade de japoma construit en prélude de l'organisation de la Coupe d'Afrque des Nations de football par le Cameroun et la quantité de compost utilisée par la municipalité de Dschang pour la mise en place et l'entretien de ses espaces verts y est associée

La production du compost de qualité a donné un regain de confiance aux agriculteurs engagés dans l'agriculture biologique. En plus des marchés de produits bio (Figure 5) organisés périodiquement dans la ville de Dschang par le Groupement d'Appui au Développement Durable (GADD), en partenariat avec la commune, une boutique de produits bio a été ouverte dans la ville de Dschang pour la vente des produits des agriculteurs formés et engagés au bio. 

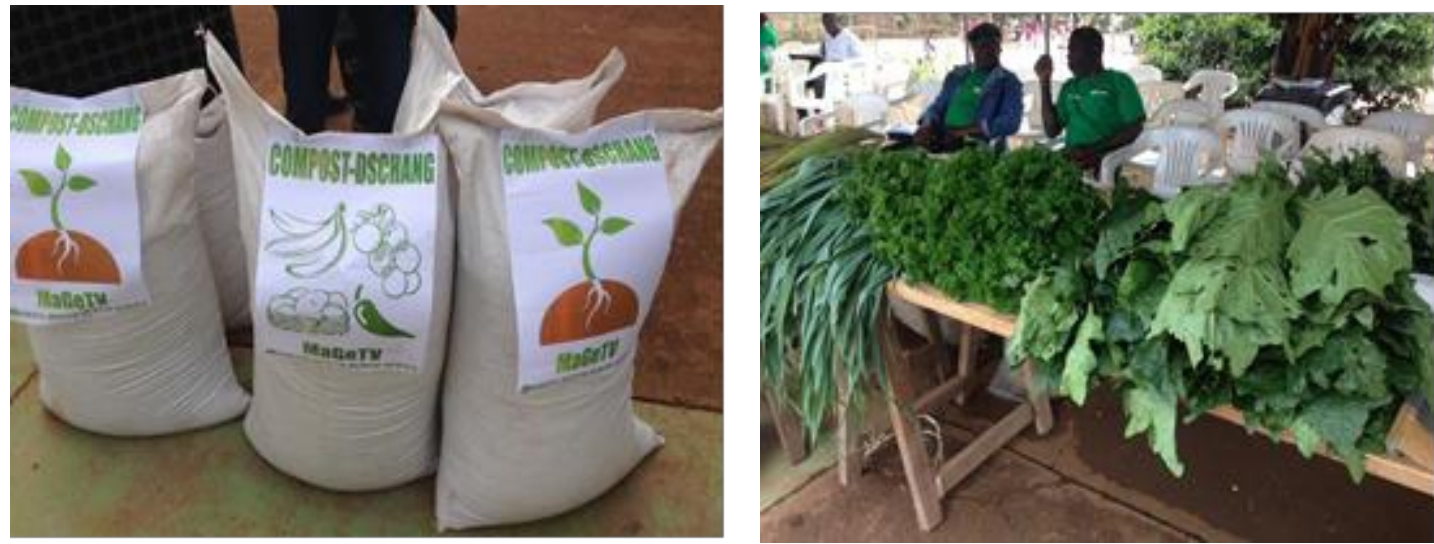

Figure 5. Expositions des produits lors d'un marché bio

Cliché Sagne Juillet 2018

\section{Développement du commerce en ligne des produits biologiques}

En plus des actions de promotion de l'agriculture à base du compost et d'organisation des marchés périodiques des produits issus à Dschang, une plateforme ${ }^{8}$ en ligne de vente des produits bio a été créée à partir de Bafoussam (moins de $60 \mathrm{~km}$ de Dschang) et les promoteurs font des descentes sur le terrain pour sensibiliser les consommateurs sur la nécessité de changer les habitudes et les producteurs sur le potentiel en termes de marché disponible pour le bio. Les producteurs qui adhèrent au réseau, s'engagent par une convention à respecter les clauses de la production et à vendre leurs produits aux promoteurs de la plateforme. De l'analyse des charges et des prix pratiqués dans l'agriculture biologique, il est ressorti que la marge bénéficiaire est comprise entre $23 \%$ et $70 \%$ en fonction de la période de l'année et du produit vendu, avec la plus grande marge bénéficiaire observée chez les revendeurs. Ceci explique sans doute la multitude d'initiatives individuelles et collectives qui voient le jour sous le label «d'agriculture bio. »

\section{Développement des sources de financement de la filière déchets}

Les sources de recettes de précollecte

Au-delà de contribuer à l'amélioration de la qualité de l'environnement dans les quartiers à accès difficile, la précollecte génère des recettes qui financent l'activité. Ainsi en 2018, pour un taux de recouvrement global moyen annuel de 65\%, l'activité a généré 5850250 FCFA de recettes pour une moyenne mensuelle de 487000 FCFA (AMGED, 2018). Suivant les conventions de délégation d'activités entre l'AMGED et les structures de précollecte, les recettes

\footnotetext{
${ }^{8}$ Le système eatbio.org est une plateforme numérique de vente en ligne et par réseau coopté promouvant la production locale et la consommation des denrées alimentaires biologiques, offrant ainsi deux possibilités d'achats aux consommateurs dont l'achat direct via le ecommerce et l'achat avec les bonus via le marketing relationnel.
} 
sont utilisées pour le financement de l'activité. Ces recettes ont permis aux structures de pré collecte en 2018 d'acheminer 2396 tricycles de déchets sur le site de compostage de Ngui correspondant à 1622 tonnes, soit l'équivalent transporté par 203 camions à compaction. En excluant les quantités de déchets collectés par coup de poing, les quantités de déchets collectés grâce aux fonds générés par la précollecte représentent 25\% des 6488 tonnes de déchets enlevés dans l'espace urbain par la collecte courante et la pré collecte en 2018 (AMGED, 2018).

\section{Les recettes de vente de compost}

Le compost de Dschang est, comme tout produit commercial, soumis au marché de l'offre et de la demande, mais surtout à celui de la concurrence. Pour faire face à la concurrence, le compost produit doit être de bonne qualité. Les analyses (Tableau 2) montrent que le compost de Dschang est bien positionné dans le marché local et régional des intrants agricoles. Il est techniquement bon et compétitif, donc accessible auprès des petits agriculteurs, mais aussi disponible en quantités pour les grandes plantations industrielles souhaitant réduire leur utilisation d'intrants chimiques. Les recettes de vente du compost produit à Dschang sont croissantes au fil du temps et la zone de chalandise initialement réduite au département de la Menoua, s'étend à d'autres régions du pays (Figure 6).

Tableau 2. Résultats d'analyse du compost de Dschang

\begin{tabular}{|c|c|c|c|c|c|}
\hline \multirow[b]{2}{*}{ PARAMETRE } & \multirow[b]{2}{*}{ UNITE } & \multirow[b]{2}{*}{$\begin{array}{c}\text { Norme } \\
\text { NFU 44-051 } \\
\text { (teneurs } \\
\text { limites) }\end{array}$} & \multirow[b]{2}{*}{$\begin{array}{l}\text { Valeurs } \\
\text { repère/cons } \\
\text { eillée pour } \\
\text { le compost }\end{array}$} & $30 / 01 / 2018$ & $14 / 05 / 2018$ \\
\hline & & & & $\begin{array}{c}\text { Compost } \\
\text { (NGUI) } \\
0 \text { mois } \\
\text { d'âge }\end{array}$ & $\begin{array}{c}\text { Compost } \\
\text { (NGUI) } 3 \\
\text { mois d'âge }\end{array}$ \\
\hline Humidité & $\%$ & & & 19,7 & 11 \\
\hline Matière sèche & $\%$ & $>\mathbf{3 0 \%}$ & & 80,3 & 89 \\
\hline $\mathrm{pH}$ & - & & $7-9$ & 9,6 & 9,2 \\
\hline \multicolumn{6}{|l|}{ Eléments Organiques } \\
\hline $\begin{array}{l}\mathrm{CO} \text { (Carbone } \\
\text { Organique) }\end{array}$ & $\% \mathrm{MS}$ & - & & 14 & 15 \\
\hline $\begin{array}{l}\text { MO (Matière } \\
\text { Organique) }\end{array}$ & $\% \mathrm{MS}$ & - & & 28,1 & 30 \\
\hline $\begin{array}{l}\text { MO (Matière } \\
\text { Organique) }\end{array}$ & $\% \mathrm{MB}$ & $>=20 \%$ & & 22,6 & 26,7 \\
\hline $\begin{array}{l}\text { N orga (Azote } \\
\text { organique) }\end{array}$ & $\% \mathrm{MS}$ & & & 0,98 & 1,21 \\
\hline \multicolumn{6}{|l|}{$\begin{array}{l}\text { Elément minéraux et } \\
\text { fertilisants }\end{array}$} \\
\hline $\begin{array}{l}\mathrm{P} \text { ass (Phosphore } \\
\text { assimilable) (P2O5) }\end{array}$ & $\mathrm{g} / \mathrm{kg}$ de $\mathrm{MB}$ & $<30$ & & 8 & 10,8 \\
\hline $\mathrm{N}$ total (Azote total) & $\mathrm{g} / \mathrm{kg}$ de $\mathrm{MB}$ & $<30$ & & 7,87 & 11 \\
\hline
\end{tabular}




\begin{tabular}{|c|c|c|c|c|c|}
\hline $\begin{array}{l}\mathrm{K} \text { (Potassium) K2O } \\
\text { (Potasse) }\end{array}$ & $\mathrm{g} / \mathrm{kg}$ de $\mathrm{MB}$ & $<\mathbf{3 0}$ & & 20,6 & 22,5 \\
\hline Mg (Magnésium) & $\mathrm{g} / \mathrm{kg}$ de MB & & & 5,5 & 6,6 \\
\hline $\mathrm{Na}$ (Sodium) & $\mathrm{g} / \mathrm{kg}$ de $\mathrm{MB}$ & & & 2,9 & 3,5 \\
\hline $\mathrm{Ca}$ (Calcium) & $\mathrm{g} / \mathrm{kg}$ de MB & & & 34,9 & 41,7 \\
\hline \multicolumn{6}{|c|}{$\begin{array}{l}\text { Rapport Carbone sur } \\
\text { Azote }\end{array}$} \\
\hline $\mathrm{C} / \mathrm{N}$ & - & $>8$ & $\begin{array}{c}>10 \&< \\
20\end{array}$ & 14,3 & 12,4 \\
\hline \multicolumn{6}{|l|}{$\begin{array}{l}\text { Eléments Traces } \\
\text { Métalliques (E.T.M.) }\end{array}$} \\
\hline Arsenic (As) & $\mathrm{mg} / \mathrm{kg}$ de MS & $<18$ & & 4 & \\
\hline Cadnium $(\mathrm{Cd})$ & $\mathrm{mg} / \mathrm{kg}$ de $\mathrm{MS}$ & $<3$ & & 1,6 & \\
\hline Chrome $(\mathrm{Cr})$ & $\mathrm{mg} / \mathrm{kg}$ de MS & $<120$ & & 66,9 & \\
\hline Mercure (Hg) & $\mathrm{mg} / \mathrm{kg}$ de MS & $<2$ & & 0,25 & \\
\hline Nickel (Ni) & $\mathrm{mg} / \mathrm{kg}$ de MS & $<60$ & & 24,5 & \\
\hline Plomb $(\mathrm{Pb})$ & $\mathrm{mg} / \mathrm{kg}$ de $\mathrm{MS}$ & $<180$ & & 176 & \\
\hline Sélénium (Se) & $\mathrm{mg} / \mathrm{kg}$ de MS & $<12$ & & 0,4 & \\
\hline \multirow{2}{*}{ Cuivre $(\mathrm{Cu})$} & $\mathrm{mg} / \mathrm{kg}$ de MS & $<300$ & & 86,8 & \\
\hline & $\mathrm{mg} / \mathrm{kg}$ de $\mathrm{MO}$ & $<600$ & & & \\
\hline \multirow{2}{*}{ Zinc (Zn) } & $\mathrm{mg} / \mathrm{kg}$ de MS & $<600$ & & 312 & \\
\hline & $\mathrm{mg} / \mathrm{kg}$ de $\mathrm{MO}$ & $<1200$ & & & \\
\hline
\end{tabular}

Source : AMGED, 2018 (Rapports d'analyse du compost de Dschang)

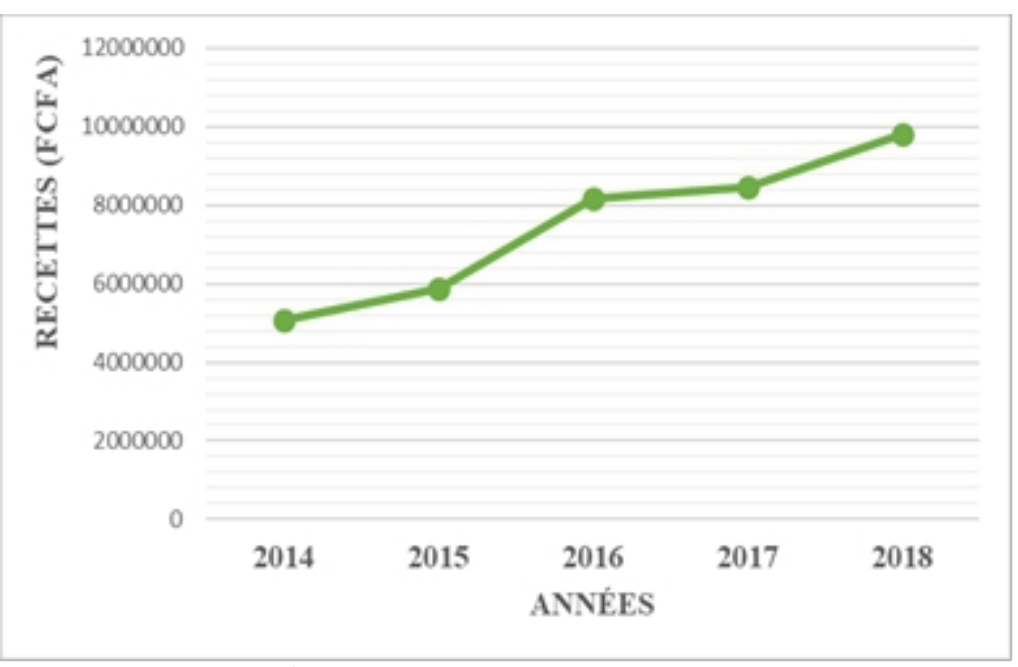

Figure 6. Évolution des recettes de vente de compost Source : Enquêtes de terrain, Juillet 2019

De 2014 à 2018, 1247 tonnes de compost, représentant 77\% de la production totale pendant la période ont été vendues, pour une recette de 37421100 FCFA. Le prix de vente a évolué au fil du temps, passant de 1500 FCFA, le sac de 50 kilogrammes à 2000FCFA. Trois catégories de clients sont définies dans la stratégie commerciale : les petits clients qui achètent moins d'une 
tonne, les clients moyens qui achètent entre une et cinq tonnes et les gros clients qui achètent plus de cinq tonnes. Les gros clients représentent $2 \%$ de l'effectif des clients, mais $60 \%$ des ventes; les clients moyens représentent $11 \%$ de l'effectif et $17 \%$ des ventes ; les petits clients représentent $85 \%$ de l'effectif des clients et $22 \%$ des ventes réalisées. Les recettes de vente de compost couvrent néanmoins environ $50 \%$ des charges de production, sans intégrer les charges de management.

\section{Les recettes de finance carbone}

Les réductions d'émissions obtenues au terme du plan de monitoring carbone sont vendues sur le marché de la compensation carbone. En vue de développer le compostage sur son territoire, la commune de Dschang a concédé la maitrise d'œuvre à une ONG nationale qui joue le rôle d'opérateur local de compostage. Une convention de financement carbone définissant les conditions de transfert et d'utilisation des fonds provenant de la valorisation des crédits carbone générés par le Projet «Valorisation des ordures ménagères de la Ville de Dschang » a été signée entre cette ONG et les organisations qui accompagnent la certification. Les subventions versées à l'ONG nationale, sont utilisées pour participer principalement à la couverture des coûts opératoires du projet et contribuent à l'équilibre financier au côté d'autres ressources, telles que les recettes issues de la commercialisation du compost, ainsi que d'autres éventuelles sources de financement. La gestion durable des déchets produit en outre des emplois verts permettant l'amélioration des conditions de vie des citadins.

\subsection{Des impacts socio-culturels contribuant à l'amélioration des conditions de vie}

Des emplois verts permettant de subvenir aux besoins de base

L’organisation internationale du travail (OIT) définit les emplois verts comme des emplois « qui aident à réduire l'impact négatifs sur l'environnement et qui débouchent à terme sur des entreprises et des économies durables d'un point de vue environnemental, économique et social ${ }^{9} »$. L'écologisation des emplois et la promotion des emplois verts, dans les secteurs tant traditionnels qu'émergents, favoriseront une économie écologiquement durable, compétitive et à faible empreinte carbone ainsi que des modes de consommation et de production durables (OIT, 2015). Au Cameroun le concept d'emploi vert est encore nouveau bien qu'il existe une variété d'emplois dont la vocation est de protéger l'environnement dans les domaines de l'assainissement (gestion des déchets), gestion des écosystèmes naturelles (forêts, aires protégés, faunes, milieu aquatiques, etc.), conception et utilisation des technologies vertes. Ces différents emplois bénéficient encore peu d'attention particulière des pouvoirs publics alors

${ }^{9}$ https://www.greengrowthknowledge.org/blog/partner-focus-international-labourorganization 
que le pays dans le cadre des accords de Paris sur le Climat en 2015 (COP 21) est engagé pour la réduction de ses émissions des gaz à effet de serre de $32 \%$ d'ici 2035. La filière déchets dans la ville de Dschang génère 80 emplois dans les différents pôles d'activité. Le pôle valorisation dispose du plus grand nombre d'employés (42), ensuite le pôle collecte (23) et le pôle précollecte (15). Les agents balayeurs de rues (plus de 10) ne sont pas inclus.

Les hommes représentent $77,5 \%$ de l'effectif des employés contre $22,5 \%$ pour les femmes. Les emplois créés sont plus ou moins stables avec $39 \%$ des employés qui exercent depuis au moins cinq ans et $37 \%$ sont affiliés à la Caisse Nationale de Sécurité Sociale (CNPS). Les employés ayant exercé plus de 10 ans dans la filière sont en charge de la collecte qui est le maillon mis en œuvre depuis toujours par la commune. $86,25 \%$ ont un salaire compris entre 30000 et 50000 FCFA ; 7,5\% ont un salaire compris entre 51000 et 150000 FCFA et $6,25 \%$ ont un salaire supérieur à 151000 FCFA. Le salaire ne constitue cependant pas la seule source de revenus pour les précollecteurs, les éboueurs de la collecte et les composteurs. Les précollecteurs ont une prime mensuelle de 25 FCFA par ménage collecté. En 2018, suivant le nombre total des ménages desservis, chaque précollecteur perçoit en moyenne 5000 FCFA de prime par mois. Les éboueurs de la collecte vendent à la fin de chaque journée de travail, les matériaux récupérés ; la moyenne des recettes de vente est de 200 FCFA/éboueur/jour. Les composteurs ont une prime de rendement semestrielle qui varie de 8000 à 15000 FCFA par agent. En cumulant les différentes sources de revenus, toute personne exerçant dans la filière déchets dans la ville de Dschang, a au moins un revenu minimum mensuel de 40000 FCFA soit 60 euros.

\section{Renforcement de la cohésion sociale}

Le renforcement de la cohésion sociale autour de la gestion des déchets est observable à plusieurs niveaux : (1) Au niveau des employés, une amicale des composteurs élargie aux précollecteurs a été créée. Cette amicale fondée sur l'entraide et la solidarité à travers les épargnes mensuels (tontines) et les travaux communautaires, a permis aux membres de réaliser des projets de construction et d'équipement de maisons d'habitation. En dehors de l'assistance mutuelle lors des évènements familiaux heureux ou malheureux, chaque membre propose annuellement une activité qui sollicitera la mobilisation du groupe, par exemple la construction de maison ou les travaux champêtres. (2) Au niveau des compostières communautaires, la mise en commun des déchets entre ménages voisins a permis de resserrer le lien social et renforcer la cohabitation. Dans un quartier, les ménages organisés autour d'une compostière ont initié des contributions par famille pour achat de matériel, et finalement ont débouché sur la mise en place d'une tontine, plus large. (3) Au niveau des agriculteurs, les rapprochements se sont matérialisés 
entre les utilisateurs de compost par la mise en place des initiatives et la création des groupes de producteurs bio.

\section{Discussion}

Les initiatives développées en matière de gestion des déchets dans la ville de Dschang concourent à la promotion d'une économie verte. Leurs impacts dans le secteur de l'environnement sont entre autres de l'amélioration de la salubrité à travers la réduction des dépôts sauvages d'ordures dans les quartiers peu accessibles, la réduction des gaz à effet de serre par compostage de la fraction fermentescible des déchets (Delarue et al., 2012) et de l'amélioration de la structure du sol par utilisation du compost (Zurbrugg \& Ahmed, 1999). Plusieurs recherches ont relevé l'impact positif de la précollecte sur l'environnement. Les travaux de Ngambi, 2015 dans la ville de Yaoundé ont montré que l'amélioration de la propreté et la restauration du paysage des quartiers ont été les premiers résultats indéniables des actions de la précollecte. L'absence de gestion des déchets génère par contre des externalités négatives encore plus onéreuses. Le Global Waste Management Outlook du Programme des Nations unies pour l'environnement (PNUE) en 2015 estime que les coûts globaux (santé, environnement) liés à la pollution des déchets déposés dans la nature ou brûlés à l'air libre s'élèvent entre 20 et 50 USD/personne/an, alors que le coût d'une gestion raisonnée reviendrait de 5 à 7 USD/personne/an (Wilson et al., 2015).

En outre, les impacts dans le secteur économique de ces initiatives sont justifiés par les recettes générées qui contribuent au financement des activités de la filière : recettes de vente du compost, recettes de précollecte issues des contributions des ménages abonnés au service. Les recettes carbones découlant de la vente des réductions d'émissions obtenues au terme du plan de monitoring carbone ont été confirmées dans les études de (Ndoumbe et al., 1995 ; Ngnikam, 2000). Les déchets issus de la consommation deviennent input dans le circuit économique et constituent un secteur économique à part entière (Meyronneinc, 1993). Plusieurs auteurs dans le cadre des recherches sur l'économie circulaire ont montré que les activités de précollecte, de récupération, de recyclage formelles ou informelles génèrent des sources de revenus aux acteurs (Ngambi, 2015 ; Mbiadjeu, 2019). L'agriculture biologique se présente comme une grande opportunité pour les pays en développement (Twarog, 2006) et le bio que nous avons utilisé dans ce travail ne traduit que ses balbutiements au Cameroun (Bayiha et al., 2019).

Par ailleurs les impacts au niveau socio- culturel, notamment le nombre d'emplois créés et le renforcement de la cohésion sociale au niveau des travailleurs ont été confirmés par Bertolini (2005). En organisant la filière déchets, la municipalité a mis en place un moyen pour intégrer des personnes 
sans formation et sans emploi, mais aussi des professionnels du domaine des déchets. La multitude des initiatives en matière de gestion des déchets dans la ville de Dschang est liée d'une part au contexte de décentralisation en cours qui nécessite la prise en compte des réalités locales et une participation des populations concernées à leurs propres aspirations. D’autre part, elle est aussi liée à la taille raisonnable de la ville et l'organisation sociale et compétitive, permettant concertation et implication des habitants, dans les solutions innovantes à leurs problèmes.

\section{Conclusion}

Le contexte de décentralisation en cours au Cameroun consacre le transfert des compétences et peu de ressources aux collectivités territoriales décentralisées. La ville de Dschang a su profiter de l'opportunité de la coopération décentralisée et des partenariats pour développer en matière de gestion des déchets, des initiatives comme la précollecte participative, le compostage et le monitoring carbone qui de par leurs impacts contribuent à la construction locale d'une économie verte. Les résultats obtenus dans la présente recherche permettent de conclure qu'on peut à travers ces initiatives changer de paradigme dans le domaine des déchets et passer du rebut à la ressource. Toutefois pour une durabilité du modèle en vue de sa pérennisation et de sa duplication dans d'autres villes, quelques pistes ont été identifiées et concernent l'implication plus active des populations dans la précollecte en s'abonnant et en payant régulièrement le service ; l'adoption du compost en agriculture par les producteurs ; l'engagement de la municipalité dans le paiement d'une redevance par tonne de déchets traités au regard des externalités positives générés par le modèle en terme d'économie des dépenses de transport du fait du compostage et la prolongation de la durée de vie de la décharge. Ceci doit être soutenu en amont par l'élaboration et la mise en place des politiques publiques favorables à l'éclosion de l'économie verte à travers la mise en place d'une gestion des déchets contribuant à la dynamique de l'économie locale et au rayonnement du territoire dans la mouvance du développement durable. Au regard des résultats obtenus, il serait important de mener des recherches sur d'autres villes moyennes ayant développées des initiatives similaires afin d'évaluer les impacts sur le triple plan environnemental, économique et social.

\section{References :}

1. ADEME (2008). Guide pratique sur le compostage. ADEME édition, Paris.

2. ADEME (2001). "Déchets organiques - Essai agronomique de plein champ d'un compost de déchets verts (résultats $8 \mathrm{e}$ année d'expérimentation)." Paris, France. 
3. Bayiha, G., Temple, L., Mathe, S. \& Nesme, T. (2019). Typologie et perspective d'évolution de l'agriculture biologique au Cameroun, Cahier Agriculture, vol 28. https://doi.org/10.1051/cagri/2019003

4. Beyeme, B.A. (2011). Influence du compost à base d'ordures ménagères sur la stabilité structurale des agrégats de sols ferralitiques rouges de Dschang. Mémoire d'ingénieur agronome, FASA, université de Dschang.

5. Bertolini, G. (2005). Economie des déchets : des préoccupations croissantes, de nouvelles règles, de nouveaux marchés, Technip, Environnement, Paris.

6. Bresson, L.M., Koch, C., Le Bissonnais, Y., Barriuso, E. \& Lecomte, V. (2001). Soil surface structure stabilization by municipal waste compost application. Soil Science Society of America Journal, vol 65, p 1804-1811.

7. Commune de Dschang (2010). Plan d'Occupation des Sols, rapport condensé.

8. Delarue, J., Flipo, B., Morizot, G. \& Tiberghien, M. (2012). Développement durable de la gestion des ordures ménagères et financements carbone : les conditions d'une mise en œuvre conjointe dans les pays en développement. Revue Sciences déchets et techniques $n^{0} 62$.

9. Kaza, S., Yao, L.C., Bhada-Tata, P. \& Van Woerden, F. (2018). What a Waste 2.0: A Global Snapshot of Solid Waste Management to 2050. Urban Development; Washington, DC: World Bank. World Bank. https://openknowledge.worldbank.org/handle/10986/30317

10. Mbiadjeu-Lawou, S. P. (2019). Quelle économie circulaire spontanée pour une ville moyenne camerounaise ? : Le cas des déchets solides ménagers de Bangangté (Cameroun). Thèse de doctorat. Cotutelle Université de Yaoundé 1, Université de Le Mans.

11. Meyronneinc, J.P. (1993). Plaidoyer pour les déchets. Apogée, Rennes.

12. Mustin, M. (1987). Le compost, gestion de la matière organique. Editions François Dubusc - Paris.

13. Naquin, P. \& Ngnikam, E. (2008). Compostage des déchets ménagers dans les pays en développement: Modalités de mise en place et de suivi d'installations décentralisées pérennes. Guide CEFREPADE, ADEME.

14. Ndoumbe N'kotto, H., Ngnikam, E. \& Wethe, J. (1995). Le compostage des ordures ménagères : l'expérience du Cameroun après la dévaluation du franc CFA. Bulletin Africain Bioressources Énergie Développement et Environnement, n 4, p 4-10.

15. Ngambi, J.R. (2015). Déchets solides ménagers dans la ville de Yaoundé (Cameroun) de la gestion linéaire vers une économie 
circulaire. Thèse de Doctorat, Université du Maine.

16. Ngnikam, E. (2000). Evaluation environnementale et économique du système de gestion des déchets solides municipaux : analyse du cas de Yaoundé au Cameroun. Thèse de Doctorat, INSA Lyon.

17. Ngnikam, E., Vermande, P. \& Rousseaux, P. (1993). Traitement des déchets urbains. Une unité de compostage des ordures ménagères dans un quartier à habitat spontané à Yaoundé - Cameroun. Cahiers Agriculture, $\mathrm{n}^{\circ} 2, \mathrm{p}$ 264-269.

18. Ngnikam, E. \& Tanawa, E. (2002). Mise en place des structures de précollecte et de traitement des déchets solides ménagères urbains dans une capitale tropicale : cas de Yaoundé. Cameroun, Yaoundé.

19. OIT (2015). Principes directeurs pour une transition juste vers des économies et des sociétés écologiquement durables pour tous, COP21

- Paris 2015.

https://www.skillsforemployment.org/edmsp1/idcplg?IdcService=GE T_FILE\&dID=353799\&dDocName=WCMSTEST4_162461\&allowI nterrupt $=1$

20. Otayek, R. (2009). Décentralisation et nouveaux acteurs locaux. Centre d'Etudes d'Afrique Noire.

21. Pagliai, M., Vignozzi, N. \& Pellegrini, S. (2004). Soil structure and the effect of management practices. Soil and Tillage Research 79, 131143.

22. Soudi, B. (2001). Compostage des déchets ménagers et valorisation du compost - cas de petites et moyennes communes au Maroc. Revue H.T.E., vol 121, p 32-56.

23. Twarog, S. (2006). Organic agriculture: A trade and sustainable development opportunity for developing countries. Trade and Environment Review, 141: 223.

24. Thonart, P., Diabate, S. I., Hiligsmann, S. \& Lardinois, M. (2005). Gestion des déchets ménagers et sites d'enfouissement technique dans les pays du Sud. Guide pratique. Retrieved from ww.iepf.org.

25. Wong, J.W.C., Ma, K.K., Fang, K.M. \& Cheung, C. (1999). "Utilization of manure compost for organic farming in Hong Kong." Bioresource Technology 67(1): 43-46.

26. Zurbrugg, C. \& Ahmed, R. (1999). Enhancing Communauty Motivation and Participation, Solid Waste Management, SANDEC News 4. 